\title{
Some Models of Reproducing Graphs: III Game Based Reproduction
}

\author{
Richard Southwell, Chris Cannings \\ School of Mathematics and Statistics, University of Sheffield, Sheffield, United Kingdom \\ E-mail: bugzsouthwell@yahoo.com \\ Received July 29, 2010; revised September 16, 2010; accepted September 18, 2010
}

\begin{abstract}
Many real world networks change over time. This may arise due to individuals joining or leaving the network or due to links forming or being broken. These events may arise because of interactions between the vertices which occasion payoffs which subsequently determine the fate of the vertices, due to ageing or crowding, or perhaps due to isolation. Such phenomena result in a dynamical system which may lead to complex behaviours, to selfreplication, to chaotic or regular patterns, or to emergent phenomena from local interactions. They hopefully give insight to the nature of the real-world phenomena which the network, and its dynamics, may approximate. To a large extent the models considered here are motivated by biological and social phenomena, where the vertices may be genes, proteins, genomes or organisms, and the links interactions of various kinds. In this, the third paper of a series, we consider the vertices to be players of some game. Offspring inherit their parent's strategies and vertices which behave poorly in games with their neighbours get destroyed. The process is analogous to the way different kinds of animals reproduce whilst unfit animals die. Some game based systems are analytically tractable, others are highly complex-causing small initial structures to grow and break into large collections of self replicating structures.
\end{abstract}

Keywords: Reproduction, Graph, Network, Game, Replication

\section{Introduction}

With the growth of the internet and digital economy, it is becoming increasingly important to understand how the way individuals interact relates to the structure of the network connecting them. This is also important in biology where the spatial distribution of individuals has a profound effect upon the evolution of the ecosystem.

Evolutionary games on networks have recently become a popular way to study these issues. Often one considers a static network within which vertices (which are thought of as players) adapt their strategies over time as they play games with their neighbours [1-3]. A question which is often asked about these systems is how does the topology of the network affect the behaviour of the players?

It is worth noting, however, that many real world networks, such as the internet, ecosystems, economies and social networks, are highly dynamic and often change in response to strategic decisions of vertices. To study this issue we propose a class of models where networks grow in a way that depends upon the strategic interactions of the vertices within. Looking at these systems we can ask a different question how does the behaviour of the players affect the topology of the network?

In [4] we introduced eight reproducing graph models within which graphs grow because their vertices reproduce. In [5] we extended these models to include the idea that vertices die of old age. In this paper (which is an elaboration of [6]) we extend these models in a different direction by incorporating game theory.

Our models are inspired by the way that animal social networks develop as individuals reproduce in the summer whilst weak individuals perish in the winter. In our models graphs change because the vertices (which have different strategies) reproduce asexually. The offspring are born with the parent's strategy and link up to their surroundings in similar way to their parents. This is rather like the way newborn animals inherit their parent's genes and environment. The vertices play games and gain payoffs as they interact with each another. Vertices 
which perform poorly get destroyed whilst successful vertices survive to further reproduce.

The systems we study can generate a rich variety of different types of dynamics. Some cases are tractable and we can derive powerful results which allow us to predict the evolution of structures under a variety of games. Other systems can generate remarkably complex dynamics involving self replicating structures. Some very simple games cause small initial structures to grow and break up into diverse collections of self replicating structures. Adaptive graph models that promote self replication have been studied before in [7], where the authors use genetic algorithms to find models that induce self replication. The difference between this work and ours is that our models were not explicitly designed to promote self replication. This fascinating behaviour arose unexpectedly as a result of our biologically inspired rules.

\section{The Models}

In each of our models a graph $G_{t}=\left(V_{t}, E_{t}\right)$ evolves over discrete time steps $t$. We think of each vertex $v \in V_{t}$ as a player which employs some strategy $\varphi(v) \in\{1,2, \cdots k\}$. The game which the vertices play is specified by a $k \times k$ payoff matrix $M$ so that $M_{i, j}$ is the payoff which an employer of the $i$ th strategy receives against an employer of the $j$ th. To obtain the graph at the next time step we update $G_{t}$ according to a two stage process.

In the first (reproduction) stage we simultaneously give each vertex an offspring vertex - which is born with its parent's strategy. The way these newborn vertices are connected up depends upon which of the eight reproduction mechanism from [4] we choose to use.

In model 0 offspring have no connections.

In model 1 offspring are connected to their parent's neighbours.

In model 2 offspring are connected to their parents.

In model 3 offspring are connected to their parents and their parent's neighbours.

In model 4 offspring are connected to the offspring of their parent's neighbours.

In model 5 offspring are connected to their parents and the offspring of their parent's neighbours.

In model 6 offspring are connected to their parent's neighbours and their parent's neighbour's offspring.

In model 7 offspring are connected to their parents, their parent's neighbours and the offspring of their parent's neighbours.

We let $R_{m}(G)$ denote the graph obtained by performing the reproduction stage upon $G$ according to the $m$ th reproduction model.
After the reproduction stage we perform the killing stage. In this stage we remove every vertex which scores a total payoff below some pre-specified 'fitness' threshold $\tau$. The total payoff $f i t(v)=\sum_{u \in N e(v)} M_{\varphi(v), \varphi(u)}$ of a vertex $v$ is the total score it gets when it plays a game with each member of its neighbourhood $\mathrm{Ne}(v)$. Sometimes we shall refer to the total payoff of a vertex as its fitness. We let $C_{\tau}(G)$ denote the graph obtained by applying the killing stage to a graph $G$ using fitness threshold $\tau$.

Our update operator $U$ is defined so that $U(G)=$ $C_{\tau}\left(R_{m}(G)\right)$ is the graph obtained by performing the reproduction stage followed by the killing stage. The graph obtained by updating $G_{t}$ is $G_{t+1}=U\left(G_{t}\right)$. In this paper we shall study how the structure $G_{t}=U^{t}\left(G_{0}\right)$ changes over time steps $t$ for different choices of initial graph $G_{0}$, game $M$, reproduction model $m$ and fitness threshold $\tau$.

The dynamics under reproduction models $0,2,4,5,6,7$ and 8 are quite tractable. Later we shall derive powerful results which allow one to predict many features of the evolution of arbitrary games under these systems. Highly complex self replicative dynamics occur when reproduction models 1 or 3 are employed. In [6] we explore this phenomenon when $m=1$. We shall begin this paper with a discussion of self replication when $m=3$.

\section{Self Replication When $m=3$}

The $m=3$ growth model is the most complicated. Even under pure reproduction [4] (where vertices are immortal) this model generates a complex structure with an interesting degree distribution [8]. When we incorporate game theory we almost immediately see complex behaviour.

\subsection{A One Strategy Case: Degree Capped Reproduction}

Even when we investigate the dynamics of one strategy games we find a system of immense complexity, which we call degree capped reproduction. In this case $\tau=-Q$, for some positive integer $Q$, and we begin with a graph $G_{0}$ where all vertices employ a strategy that scores a payoff of -1 against itself. Updating a graph $G_{t}$ under the degree capped reproduction model consists of performing two stages.

Stage 1, Reproduce: simultaneously give every vertex of $G_{t}$ an offspring vertex which is connected to its parent and its parent's neighbours.

Stage 2, Kill: remove every vertex of degree greater than $Q$. 
Under degree capped reproduction graphs tend to grow and break into other graphs. Phase diagrams represent how connected structures produce one another using arrows. The arrows pointing out of a graph point towards the connected graphs produced by updating it. The numbers on the arrows correspond to the numbers of such graphs produced.

When we evolve an isolated vertex under degree capped reproduction with $Q=4$ we get the dynamics depicted in Figure 1. The structure grows up over the first two updates and then breaks into four more isolated vertices. These vertices continue to replicate in a similar way.

The way the isolated vertex evolves when $Q=5$ is considerably more elaborate Figure 2. In this case the structure grows over the first four updates to become a square structure with four 'arms'. This structure breaks into four smaller structures which replicate themselves and other self replicating structres. Eventually more isolated vertices are generated and one is left with a soup of eleven different kinds of self replicating graphs.

As $Q$ increases the self replicative behaviour becomes increasingly intricate. Figure 3 shows the self replicative habits of the 13 different graphs that grow from an isolated vertex when $Q=7$.

Increasing $Q$ further causes an immense increase in complexity. When $Q=10$, the isolated vertex eventually generates an ecosystem of 121 different kinds of self replicating structures. These structures produce copies of one another according to a large phase diagram similar to Figure 7. This behaviour is incredibly complicated, many of the structures produced have hundreds of vertices. Figure 4 shows an example.
There is much more to study about degree capped reproduction. We conjecture that every finite initial condition generates a bounded number of different self replicating structures under finite $Q$. A method to prove this conjecture or identify the self replicating structures would be highly desirable. However the rapid increase in complexity with $Q$ makes it doubtful that such a method can be found.

Degree capped reproduction can be thought of as a toy model for the way an animal social network changes over time. Consider a set of reproducing individuals which share some vital resource, like food. When new offspring are born they will share resources with their parents and their parent's associates. When an individual shares its resources with too many other individuals it starves and dies.

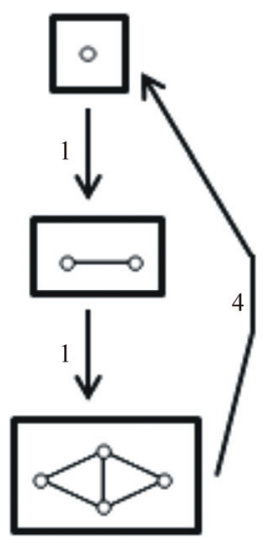

Figure 1. A phase diagram showing how the different structures that grow from an isolated vertex generate each other when $Q=4$.

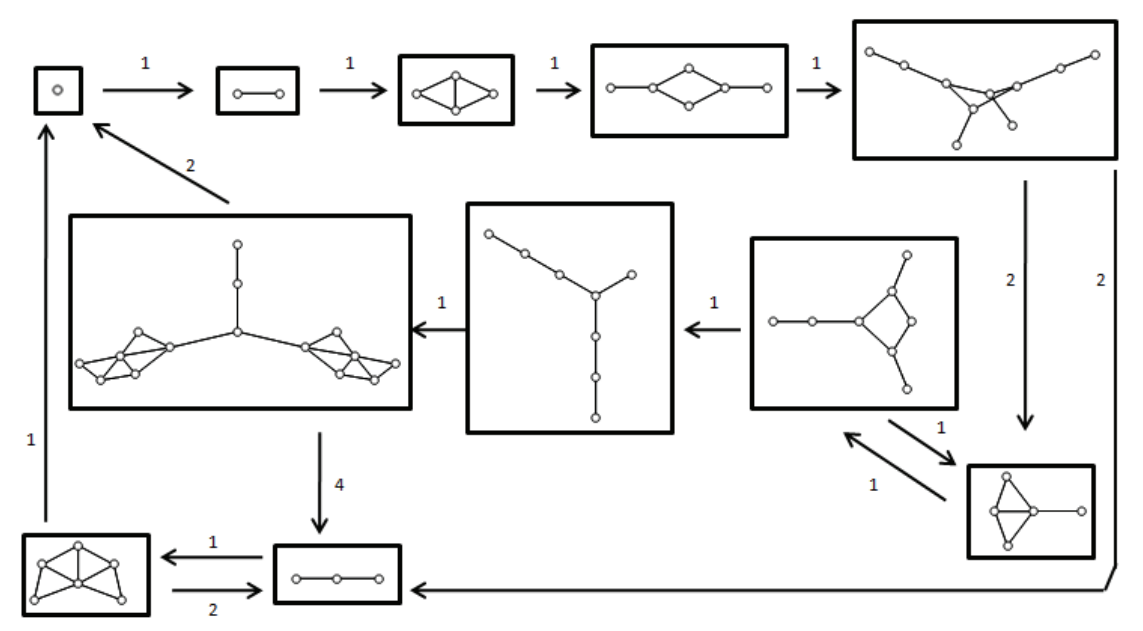

Figure 2. A phase diagram showing how the different structures that grow from an isolated vertex generate each other when $Q=5$. 


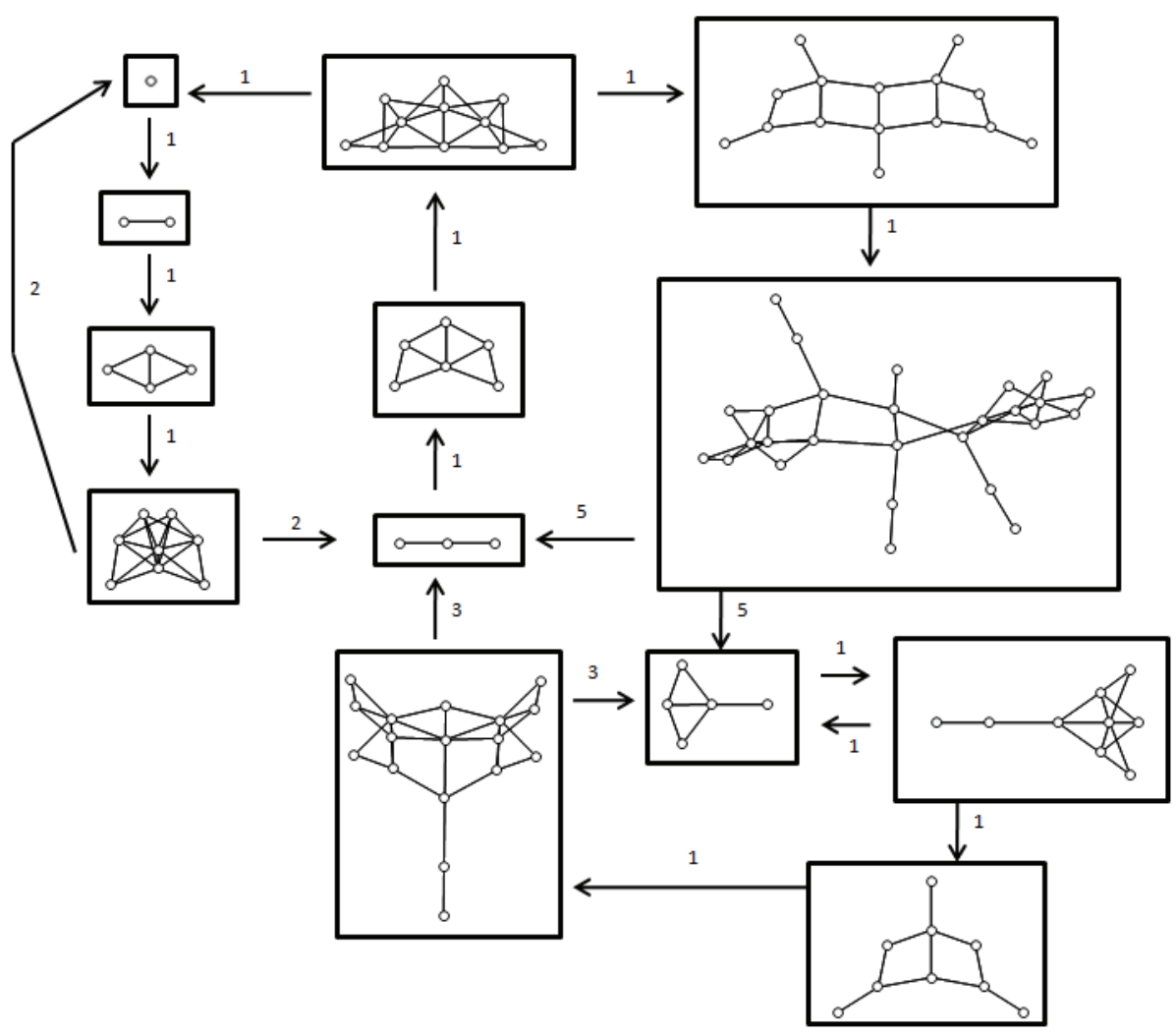

Figure 3. A phase diagram showing how the different structures that grow from an isolated vertex generate each other when $Q=7$.

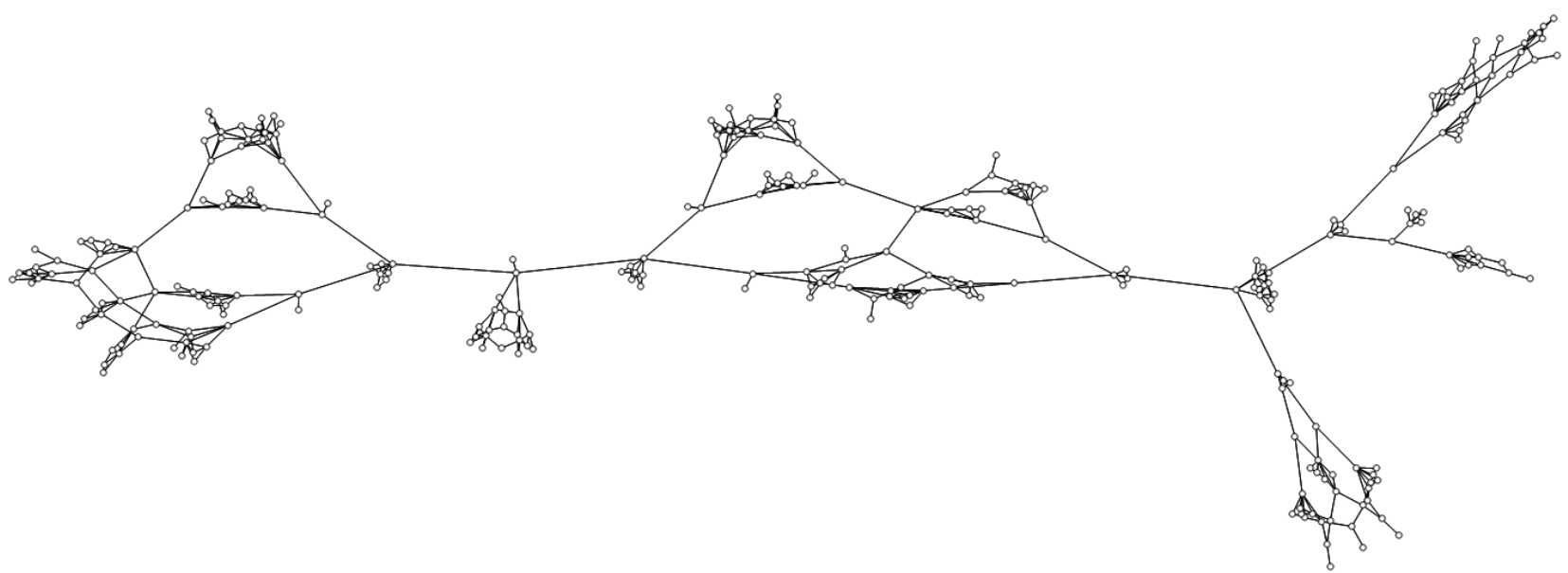

Figure 4. One of the 121 self replicating structures that get produced when one evolves an isolated vertex with $Q=10$. This graph has 292 vertices.

It is remarkable how these simple biologically inspired rules can give rise to such a diverse collection of self replicating structures. It suggests that complex self replicating structures, like life, do not have to be the result of complex rules. Self replication has been achieved by other decentralised systems, like cellular automata, but in our systems we see self replicating structures that can diversify their forms. If we extended our models to include some external forces which destroy vertices based upon local topology it seems 
likely our structures will 'evolve' towards self replicating forms that suit their external environment.

\subsection{An Example with Two Strategies}

Many other types of behaviour can be produced when reproduction model 3 is used with other games. Consider, for example, the case where $\tau=-12$ and the game is specified by the payoff matrix

$$
\left(\begin{array}{cc}
-3 & 2 \\
0 & -5
\end{array}\right)
$$

When we run this system, starting with the graph shown in Figure 5, we see a large structure grow up that contains employers of both strategies. The large structure seems to grow relentlessly whilst throwing off mono strategy fragments that self replicate in accordance with degree capped reproduction.

This coexistence of self replicating structures on different scales also occurs in biological processes. As large organisms grow they house many micro organisms which compete and reproduce. Occasionally these micro organisms may leave the body of their host to self replicate elsewhere.

There is much more to study about the dynamics of games when $m=3$. It appears as though when $\tau \geq 0$ the dynamics are relatively constrained. In cases like this structures seem to either die away or reach a regime of unrelenting growth without structural disconnections. We have made a brief exploration of game dynamics when $\tau<0$ but many mysteries remain. Are there any games which cause the number of vertices to fluctuate chaotically over time? Does there exist a game which generates a computationally universal system (in that arbitrary computations can be emulated by evolving appropriate initial conditions)?

\section{The Dynamics When $m=1$}

Model 1 is the other growth mechanism that can produce highly complex behaviour. A vertex $v$ will survive the $U$ update when $m=1$ if and only if $f i t(v) \geq \frac{\tau}{2}$ whilst $v$ 's offspring will be present after the $U$ update if and only if $f i t(v) \geq \tau$.

The complexity of $U^{t}(G)$ 's dynamics depends largely upon the sign of the fitness threshold $\tau$. When $\tau=0$ the dynamics are relatively easy to describe, structures either grow forever or fizzle away to nothing. When $\tau>0$ it seems (although we cannot prove) that connected structures either grow forever, fizzle away, or become fixed. When $\tau<0$ dynamics of enormous complexity can ensue.

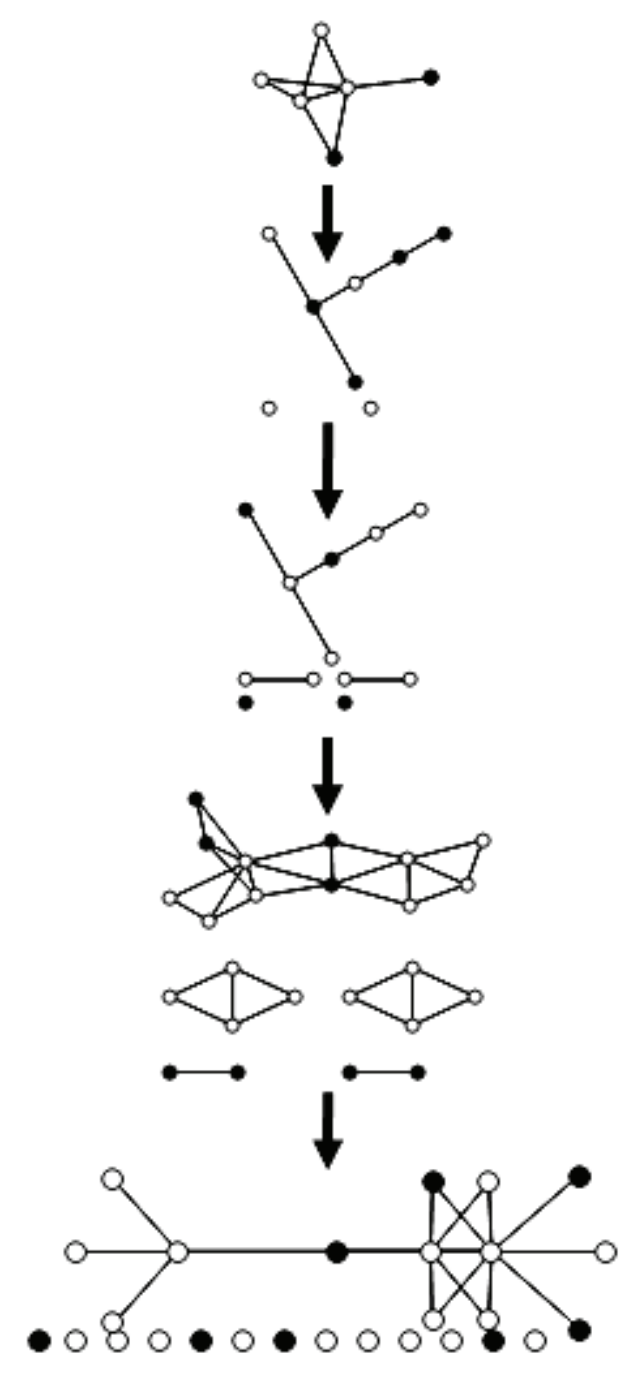

Figure 5. The evolution of an example structure when $\tau=-12$ and the game is as defined in Subsection 3.2. The arrows denote update operations (which may lead to structures breaking into pieces). Employers of strategies 1 and 2 are represented by white and black vertices respectively.

\subsection{The Case When $\tau=0$}

The easiest case is when $\tau=0$; in this case we have

$$
U(G)=\left(C_{0} \circ R_{1}\right)(G)=\left(R_{1} \circ C_{0}\right)(G) .
$$

where $f \circ g$ denotes the composition of functions $f$ and $g$.

Swapping round the order of killing and reproduction allows us to gain the expression

$$
U^{t}(G)=\left(C_{0} \circ R_{1}\right)^{t}(G)=\left(R_{1}^{t} \circ C_{0}^{t}\right)(G) .
$$

So in other words $U^{t}(G)$ is the graph obtained by taking $G$ and applying the killing operator to it $t$ 
times and then applying the reproduction operator to the resulting graph $t$ times.

A vertex $v$ in $G$ will reproduce forever under $U^{t}$ if and only if it does not get removed by the $C_{0}^{\infty}$ operation. This means every finite graph will either die out or reach a regime of unbounded growth.

\subsection{The Case When $\tau>0$}

When $\tau>0$ it seems that the long term behaviour of $U^{t}(G)$ is again quite restricted. We conjecture that any graph $G$ will evolve under $U^{t}$ towards either destruction, unbounded growth or some mixture of fixed subgraphs and subgraphs that grow forever (in that none of their descendants ever die). Computer simulations are our main reason for believing this however we can demonstrate some restrictions when $M$ is non-negative.

When $\tau>0$ and $M$ has no negative entries then for any initial graph $G$ we will have

$$
R_{1}^{t} \circ C_{\tau}^{t}(G) \subseteq U^{t}(G) \subseteq R_{1}^{t} \circ C_{\frac{\tau}{2^{t}}} \circ C_{\frac{\tau}{2^{t-1}}} \circ \cdots \circ C_{\frac{\tau}{2}}(G) .
$$

Thus vertices of $G$ that get removed by the

$$
C_{\frac{\tau}{2^{t}}} \circ C_{\frac{\tau}{2^{t-1}}} \circ \cdots \circ C_{\frac{\tau}{2}}
$$

operation, as $t \rightarrow \infty$, will certainly not enter a regime of unrestricted reproduction. On the other hand vertices that survive the $C_{\tau}^{t}$ operation, as $t \rightarrow \infty$, will surely reproduce forever. Somewhere between these two extremes are vertices that become infertile and immortal (i.e. static). When all vertices are static the graph will be fixed under the $U$ update; this occurs when each vertex has a fitness in $\left[\frac{\tau}{2}, \tau\right)$.

\subsection{The case when $\tau<0$}

When $\tau<0$ exotic dynamics can occur. In addition to the null, fixed and unbounded growth behaviour types that we found before, when $\tau<0$, we encounter self replicative dynamics similar to those discussed in section 3 ([6] is largely devoted to the study of this phenomena). In [6] we made an extensive investigate of the dynamics of degree capped reproduction when individuals reproduce according to the $m=1$ model (i.e. when offspring are not connected to their parents).

The dynamics of degree capped reproduction when $m=1$ are similar to those when $m=3$. When $Q$ is small structures tend to generate a reasonably small collection of self replicating structures. Figure $\mathbf{6}$ shows the structures that get generated when one evolves a single edge under this system with $Q=4$.

Again as one increases $Q$ one sees a dramatic increase in the complexity of the self replicative processes. Figure 7 shows a phase diagram which illustrates how the 210 self replicating structures that get made by evolving a single edge produce each other.

In [6] we demonstrate how this self replicative behaviour persists when one adds a degree of randomness into the update procedure. In this paper we also describe many two strategy games that can promote self replication.

\section{The Dynamics When $m=2$}

The dynamics of $U^{t}(G)$ when $m=2$ are very similar to dynamics of reproduction model 2 in the age capped systems [5]. Offspring are born only connected to their parent and so repeated updates cause tree structures to sprout out of the initial graph structure (which may itself disintegrate). The fundamental form of the tree that will grow out of a vertex employing a given strategy is just

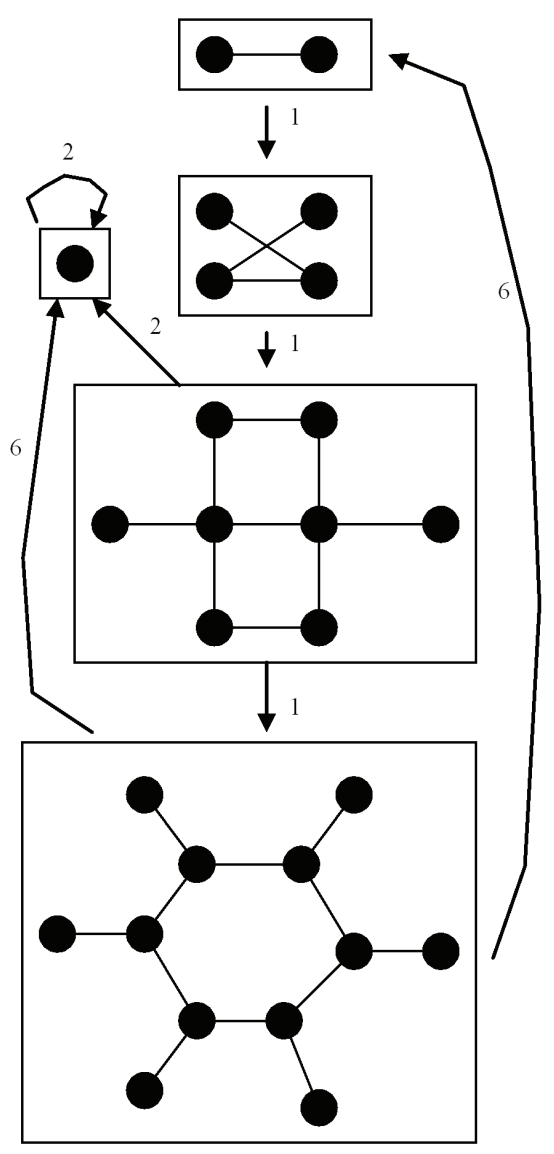

Figure 6. A phase diagram showing the self replicating structures which emerge from evolving a single edge when $Q=4$ and $m=3$. 


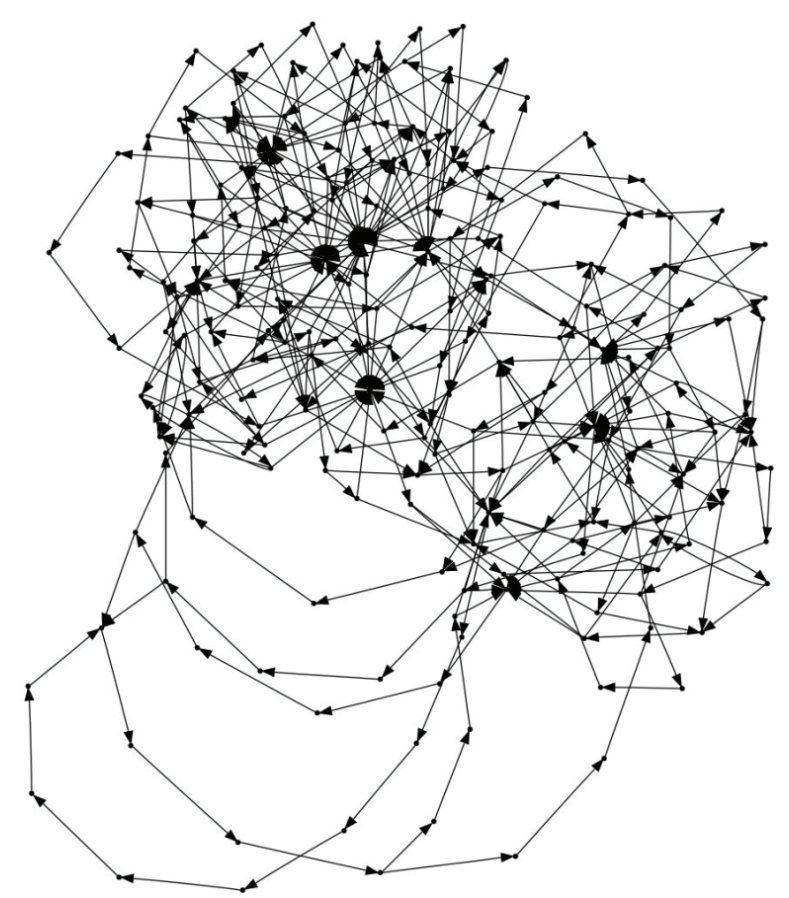

Figure 7. A phase diagram showing the self replicating structures which emerge from evolving a single edge when $Q=8$ and $m=3$. The nodes represent connected graphs (many of which hold thousands of vertices). We have neglected to number the edges or consider the isolated vertex (which many graphs produce).

dependent upon how the vertex develops in isolation (a vertex's structural surroundings does not influence its descendant's dynamics).

Suppose our initial graph $P$ is an isolated vertex that employs a strategy that receives a payoff $s$ against itself.

When $s<\tau$ our graph will not be fit enough to make it past the first killing stage; in this case we will just have that $U(P)$ is the empty graph.

When $s \geq \tau$ and $s \geq 0$ we will have $U^{t}(P)$ $=R_{2}^{t}(P)$ at $t$, so our graph will grow forever as it does in our pure reproduction model [4].

When $s \geq \tau$ and $s<0$ our tree will grow as normal until the degree of the original vertex gets too large and our tree breaks into several smaller trees. Updating within this scenario is equivalent to giving every vertex an offspring connected to its parent and then removing every vertex of degree $>\frac{\tau}{s}$. Our graph will replicate itself under this regime in a similar way to the systems we encountered in [5].

Under this regime any connected component of the form $R_{2}^{k}(P)$ with $k \in\{0,1, \ldots,\lfloor\tau / s\rfloor-1\}$ will get updated to become $U\left(R_{2}^{k}(P)\right)=R_{2}^{k+1}(P)$. On the other hand when we update the graph $R_{2}^{\lfloor\tau / s\rfloor}(P)$ with $U$ it breaks into a set of connected components. This set holds two disjoint copies of $R_{2}^{k}(P)$, for each $k \in\{0,1, \cdots$, $\lfloor\tau / s\rfloor-1\}$, and nothing else.

This new structure will grow and break up in a similar way to the original and so we may use the theory of Leslie matrices to describe the asymptotic distribution of the different structures in a similar way to in [5].

\section{The Dynamics When $m=4$}

When $m=4$ we have that $U(G)$ is the graph obtained by taking two disjoint copies of $G$ and then removing every vertex of fitness less than $\tau$. After repeated updates $U^{t}(G)$ will just consist of $2^{t}$ disjoint copies of $C_{\tau}^{t}(G)$.

\section{The Dynamics When $m=5$}

Updating a graph when $m=5$ consists of giving every vertex an offspring that is connected to its parent's neighbours and the new offspring of its parent's neighbours, then removing each vertex of fitness below $\tau$. Thanks to the fact that each vertex is automorphically equivalent to its offspring in $R_{5}(G)$ we can perform a trick that gives a relatively simple description of $U^{t}(G)$ 's dynamics.

Applying the $R_{5}$ update will cause each vertex $v$ to yield a parent vertex and a child vertex which will both have double the fitness that $v$ had before. This means that

$$
U(G)=C_{\tau} \circ R_{5}(G)=R_{5} \circ C_{\frac{\tau}{2}}(G)
$$

which leads us to the fact that

$$
U^{t}(G)=R_{5}^{t} \circ C_{\frac{\tau}{2^{t}}} \circ C_{\frac{\tau}{2^{t-1}}} \circ \cdots \circ C_{\frac{\tau}{2}}(G) .
$$

In other words $U^{t}(G)$ is the graph obtained by taking $G$ and removing all the vertices that have fitness less than $\frac{\tau}{2^{1}}$ then removing all the vertices that have fitness less than $\frac{\tau}{2^{2}} \ldots$ and so on ... then removing all the vertices that have fitness less than $\frac{\tau}{2^{t}}$ then taking the resulting graph and applying the $R_{5}$ growth operator to it $t$ times.

This gives us a way to quickly determine the fate of descendants of initial vertices. If a vertex survives the

$$
C_{\frac{\tau}{2^{t}}} \circ C_{\frac{\tau}{2^{t-1}}} \circ \ldots \circ C_{\frac{\tau}{2}}
$$

operation, as $t \rightarrow \infty$, then its descendants are destined to multiply forever. If an initial vertex does not survive this killing operation then its descendants are destined to 
eventually die out. This allows us to predict the dynamics of our models with much less effort. It also implies that the rich self replicative dynamics observed when $m=1$ and $m=3$ cannot occur under this model.

\section{The Dynamics When $m=6$}

Updating a graph $G$ under the $m=6$ model consists of making an 'offspring copy' of $G$, linking each vertex with its offspring in the offspring copy, and then removing every vertex that has fitness below $\tau$. The symmetry between parents and their offspring allows us to do a similar trick to that used to simplify the $m=5$ case.

Let $C_{n, \tau}$ be defined such that for any strategy attributed graph $G$ we have that $C_{n, \tau}(G)$ is the graph obtained by taking $G$ and removing each vertex $v$ such that $f i t(v)+n \cdot M_{\varphi(v), \varphi(v)}<\tau$.

After the $R_{6}$ update both the offspring and the surviving parent will have the fitness of their original vertex plus the score that their strategy gets against itself (the effect of the 'umbilical connection' between parent and offspring). This means

$$
U(G)=C_{0, \tau} \circ R_{6}(G)=R_{6} \circ C_{1, \tau}(G)
$$

from which it follows that

$$
U^{t}(G)=R_{6}^{t} \circ C_{t, \tau} \circ C_{t-1, \tau} \circ \cdots \circ C_{1, \tau}(G) .
$$

This means that again one can say in advance whether the descendants of initial vertices will die out or multiply forever.

\section{The Dynamics When $m=7$}

Updating a graph with $m=7$ consists of giving every vertex an offspring with the same strategy that is connected to its parent, its parent's neighbours and the new offspring of its parent's neighbours, and then removing every vertex of fitness less than $\tau$. Again symmetry allows us to do a trick that gives us a simpler view of the dynamics.

For any vertex $v$ of fitness $f i t(v)$ in $G$ both $v$ and $v$ 's offspring within $R_{7}(G)$ will have fitness 2. $f i t(v)+M_{\varphi(v), \varphi(v)}$. Let

$$
h_{y}(x)=2 x+y
$$

and let

$$
C_{r, \tau}^{*}(G)
$$

be the graph obtained by taking $G$ and removing every vertex such that

$$
h_{M_{\varphi(v), \varphi(v)}^{r}}^{r}(f i t(v))<\tau
$$

In this case we will have that

$$
U(G)=C_{0, \tau}^{*} \circ R_{7}(G)=R_{7} \circ C_{1, \tau}^{*}(G)
$$

which means that

$$
U^{t}(G)=R_{7}^{t} \circ C_{t, \tau}^{*} \circ C_{t-1, \tau}^{*} \circ \cdots \circ C_{1, \tau}^{*}(G)
$$

so again we can determine the fate of our initial vertices descendants without explicitly running the system.

\section{Conclusions}

In this paper we have investigated many features of game theory based reproducing graph models. Many kinds of behaviour can be generated and there are many perspectives through which one may view these systems.

From an evolutionary game theory standpoint these systems are interesting because they provide a (possibly analytic) method to investigate how the outcome of games effects the formation of communities. To investigate this it would be useful to compare the dynamics of our graphs with the behaviour of other systems based upon game theory.

From the perspective of theoretical biology and artificial life these systems are interesting because they can demonstrate rich self replicative behaviour. The existence of these systems begs the question of what other adaptive graph models can do. Finding other systems with similar behaviour might be useful for modeling systems in biology and inventing nanotechnology.

From the perspective of complex systems research these systems are interesting because they can generate highly complex behaviour from simple initial conditions. An interesting question from this perspective is just how complicated our systems are. Is there a simple formula to describe the dynamics of graphs under degree capped reproduction? Are features of their dynamics formally undecidable?

Taking a more practical perspective one might ask what real world applications these systems may have. Using game theory to reward or punish graphs for growing in particular ways could be an interesting way of designing networks for specific purposes. It would be interesting to see if one could use systems similar to those we have discussed for design of communication networks or self repairing structures.

\section{Acknowledgements}

The authors gratefully acknowledge support from the EPSRC (Grant EP/D003105/1) and helpful input from Dr Jonathan Jordan, Dr Seth Bullock and others in the 
Amorph Research Project (www.amorph.group.shef.ac. uk).

\section{References}

[1] M. Nowak and R. May, "Evolutionary Games and Spatial Chaos," Nature, Vol. 359, No. 6398, 1992, pp. 826-829.

[2] R. Southwell and C. Cannings, "Best Response Games on Regular Graphs," Under Review, 2010.

[3] F. Santos, J. Pacheco and T. Lenaerts, "Evolutionary Dynamics of Social Dilemmas in Structured Heterogeneous Populations," Proceedings of the National Academy of Sciences of the United States of America, Iowa, Vol. 103, 2006, pp. 3490-3494.

[4] R. Southwell and C. Cannings, "Some Models of Repro- ducing Graphs: I Pure Reproduction," Applied Mathematics, Vol. 1, No. 3, 2010, pp. 137-145.

[5] R. Southwell and C. Cannings, "Some Models of Reproducing Graphs: II Age Capped Vertices," Applied Mathematics, Vol. 1, No. 4, 2010, pp. 251-259.

[6] R. Southwell and C. Cannings, "Games on Graphs that Grow Deterministically," Proceedings of the 1st International Conference on Game Theory for Networks (GameNets), Istanbul, 13-15 May 2009.

[7] H. Tomita, H. Kurokawa and S. Murata, "Automatic Generation of Self-Replicating Patterns in Graph Automata," International Journal of Bifurcation and Chaos, Vol. 16, No. 4, 2006, pp. 1011-1018.

[8] J. Jordan and R. Southwell, "Further Properties of Reproducing Graphs," Under Review, 2010. 